sion the importance of dentistry as a profession than by re quiring that the education of the candidates for the dental degree be raised. Dr. Power feared that the mission of a college sometimes is misinterpreted. The mission of every pro. fessional school, he thought, consists in giving to a graduate the least possible amount of knowledge that can be possessed, in order that he may be allowed to practice with safety to the public. One lifetime is too short for a dental or a medical school even to pretend to familiarize a man with every form of disease he may be called on to treat in future years. The diploma represents the acquisition of the fundamentals on which the knowledge of dentistry is based. When dentists look at the matter in that light, when they will see things as they really are, the elevation of dentistry as a profession will commence, and dentists will be in a position to demand the recognition which they are now seeking. In conclusion Dr. Power referred to the mission of the profession itself. He believes that dentists should exercise the greatest care and practice along scientific lines. They should realize that they have reciprocal obligations to each other, and that the whole professinn is affected by the words and actions of the indiridual. They should do everything they can to assist in its elevation. Professional ethics, social bearing and high moral culture will do most for dentistry, most for humanity, and impress the medical profession, the public, and all concerned with the facts that the dental profession deserves proper recognition.

Dr. JAMEs McMAves, Hartford, Comn., called attention to the fact that of the 35,000 dentists in the country, a large proportion of whom are college graduates, only a few are mem. hers of the Section on Stomatology. He does not believe that dental colleges are as poor some say they are. He believes that every dental college in the country graduates each year men who are the equal, in their line, of any medical graduates, and that, as a rule, the dental graduate is better fitted to go out and practice his profession than is the medical graduate. His fingers are elucated, his opportunities in clinies are greater, and when it comes to the use of anesthetics he has a practical knowledge that the average young medical student does not have. What is the trouble? he asked. When the dental graduate goes out to practice he stops right there; he does not seem to study; he does not seem to take any interest in societies. They do not seem to take any interest in their profession; they do not seem to care to study. Nor do dentists buy books. Dr. McManus thought that the trouble with the dentists has been that when they got where they were legally allowed to practice, they joined this organization and that organization, and they spend their money for almost every cause except the cause of dentistry. Dentistry, as a majority of dentists practice it, is a calling. A feiv dentists are professional men, and some of these have the degree of M.D. Those who have not this degree, Dr. McManus believes, are as good professional men as are those who have it. The dental graduate, he said, is well educated, or at least he has the opportunity to get a good education, for most every dental college gives that, and it is his own fault if, after he leaves college, he does not become better educated.

Dr. Truman W. Brophy, Chicago, thought that the reference made to the shortcomings of the schools of medicine, regarding their failure to teach many of the diseases and injuries of the oral eavity, is generally recognized, but he urged that the matter be taken up in the Section on Medicine, where it properly belongs. He felt that the trend of the papers and of the general discussion suggested that the word mechanics is an objectionable one, and that it should not be given the weight and consideration that it is receiving by dental colleges and dentists generally. It must be remembered, however, he said, that among the greatest men the world has produced have been those who attracted attention not only by their genius, but also by their digital skill. Men such as Michael Angelo and Thorwaldsen were mechanies, therefore dentists must not forget the achievements and the possibilities of the mechanical side of their profession. Dr. Brophy agreed with Dr. McManus that what is needed is men of education in dentistry. A man will stand for exactly what be is worth. If he is cultured, if he has education, if he has capacity, such qualities will be recognized, always. It docs not make any difference what titles a man has; he will be recognized for what he knows and what he is able to do. As to dentists, they need not appeal to the medical profession for recognition.

Dr. F. L. Fossume, New York City, emphasized the necessity of the dentist to fortify himself with a knowledge of pathology and histology, so that he can discuss all dental lesions in terms acceptable to medical men. Every man, he said, will ret the recognition he deserves if he works, and if he pays attention to the opportunities that are offered of practicing dentistry on a high plane and according to a high standard. He believes that stomatology can stand alone and that the dental profession is insufficiently educated in pathology and histology. The cases that do not yield to treatment, those with lesions around the teeth and their sockets, are almost analogous to cases of gallstones which are treated therapeutically. Every physician, failing to secure results, will immediately proceed to examine the patient's urine, and thus arrive at a safe conclusion as to the etiologic causes of the disease and the reason for his failures. The ordinary dentist, practicing dentistry more or less mechanically and using only the fundamental principles of dentistry, is not doing justice to his patients when he fails to go a little deeper into the causes than merely to say, "This case is incurable." Dr. F'ossume thought that if the Section on Stomatology could unite with some other section it would be a great education for dentists and a grand opportunity to put the entering wedge into the collateral profession and gain its respect and confidence. Physicians do not realize what dentistry of to-day is. Modern dentistry is as different from what it was in its fundamental principles as any of the other sciences are in their fundamental principles, which is une to scientific research work, done by men who have deroted their time and their money to research along these lines.

\section{SUBPHRENIC ABSCESS AS A COMPLICATION OF APPENDICITIS.*}

$$
\begin{gathered}
\text { DANIEL N. EISENDRA'TH, A.B., M.D. } \\
\text { CHICAGO. }
\end{gathered}
$$$$
111
$$

\section{HISTORICAL DATA.}

The first accurate description of a subphrenic abscess was published by Bouchard ${ }^{1}$ in $186 \%$. In 1882, Leyden ${ }^{2}$ reported several cases in which the diagnosis was made clinically. Maydl, ${ }^{3}$ in 1894 , collected 179 cases, which he divided into tweive groups, according to their cause. Among these were 23 following appendicitis. Since this time Weber, ${ }^{4}$ Sachs $^{5}$ and Elsberg ${ }^{6}$ have reported a number of cases. Elsberg was able to collect 73 cases of subphrenic abscess following appendicitis, which had been published up to 1901. I have been able to add 33 cases, including 5 of my own.

\section{FREQUENCY.}

Few statistics are available from which to draw deductions as to the relative frequency of this complication. Weber found it nine times in 350 cases, and Moschowitz found 8 cases of subphrenic abscess among 2,000 cases of appendicitis occurring in Mt. Sinai Hospital.

\section{ANATOMY AND PATHOLOGY.}

The subphrenic region is divided into a right and left space by the falciform ligament of the liver. An ab-

- Read before the Chicago Medical Society, Oct. 9, 1907.

1. Bull. de la Soc, Anatomique, 1862 .

2. Ztscbr. f. klin. Med., 1882 .

3. Subphrenische Abscesse, Josef Safar, Wien, 1894.

4. Deutsche Ztschr. f. Chir., liv.

5. Arch. f. klin. Chir., l.

6. Ann. of Surgery. $x x x i v$.

7. Arch. f. klin. Chir., Ixxxil 
scess situated in the right space does not communicate with the left, unless the septum between the two is broken down. Each subphrenic space is again divided, incompletely, into an anterior and posterior space by the coronary ligament. A portion of the right lobe of the liver is not covered by peritoneum, owing to the separation of the two layers of the coronary ligament. This area is about the size of the width of the palm of the adult hand, but it plays no rôle in the pathology of subphrenic abscess. The right lobe of the liver lies in almost direct contact with the lower portion of the bony thorax, being separated from it by the diaphragm and the costophrenic sinus of the pleura.

Subphrenic abscesses may be located within the peritoneal cavity or in the retroperitoneal tissues. If intraperitoneal, the abscess may only occupy a small portion of either subphrenic space, either laterally or in

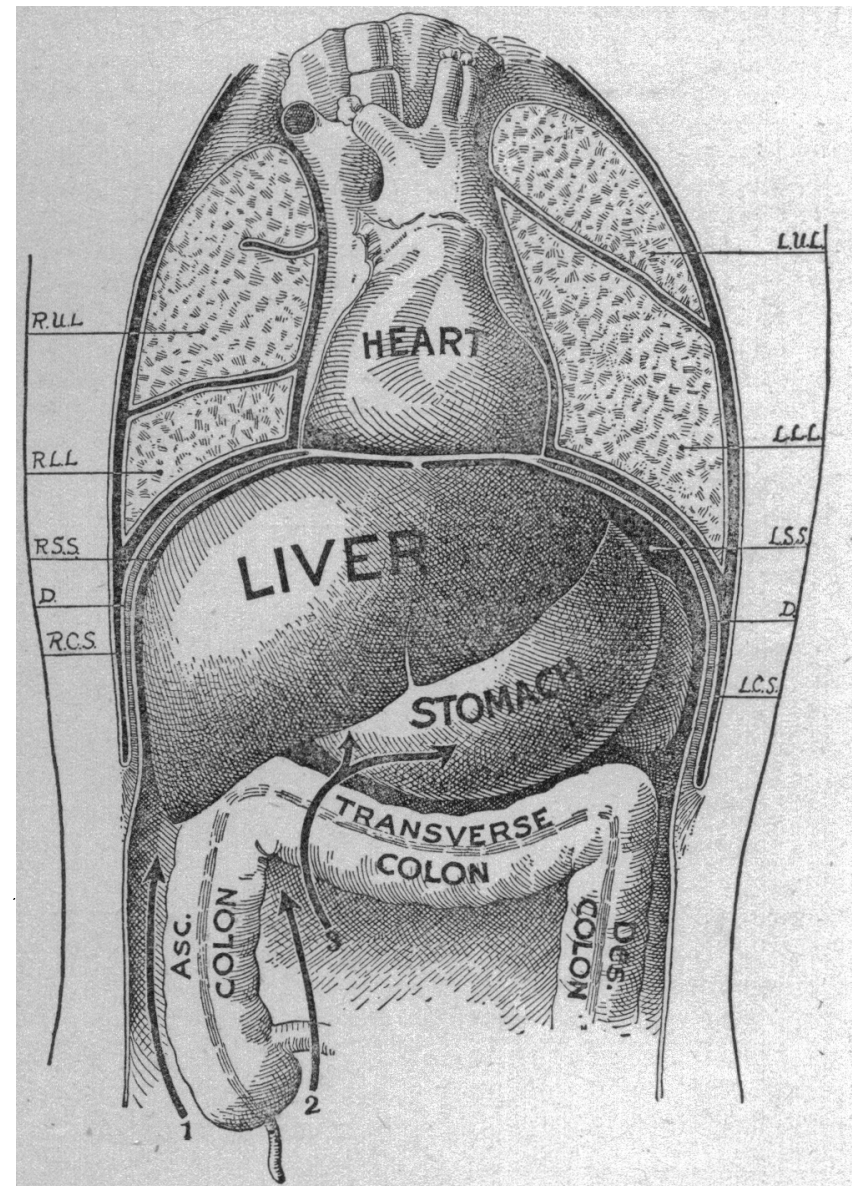

Fig. 1.-Modes of transmission of infection to right and left subphrenic spaces from appendix region. 1. Along outer side of colon. 2. Along inner side of colon. 3. Across transverse colon and stomach. The double arrow 3 shows that infection can either and stomach. The double arrow 3 shows that infection can either travel in front of the stomach and cause an abscess in the general peritoneal cavity between the spleen and liver, or the infection can
pass through the foramen of Winslow into the Iesser peritoneal cavity. R. U. L., right upper lobe of lungs. R. L. L., right lower lobe. R. S. S., right subphrenic space. D., diaphragm. R. C. S. right costophrenic sinus. $L$. U. L., left upper lobe of lungs. $L$. L. L., left lower lobe. L. S. S., left subphrenic space. L. C. S., left costophrenic sinus.

front or behind. The abscess may be located high up under the dome of the diaphragm. It rarely fills the entire right subphrenic space, being usually limited by adhesions between the liver and the inferior space of the diaphragm.

The intraperitoneal variety is far more common after appendicitis than the extraperitoneal. In the latter the infection travels upward behind the ascending colon in the retroperitoneal tissues. It can not extend very high upward, owing to the close attachment of the peritoneum to the inferior surface of the diaphragm. In the intraperitoneal variety (Fig. 1) infection either travels along the inner or outer side of the colon, or between its anterior aspect and the abdominal wall. In the 106 cases which I have been able to collect, the abscess was intraperitoneal in 50 per cent., and extraperitoneal in 25 per cent. In the remaining reports the location was not given or was doubtful.

A subphrenic abscess may follow an attack of appendicitis in which there has been no suppuration in or

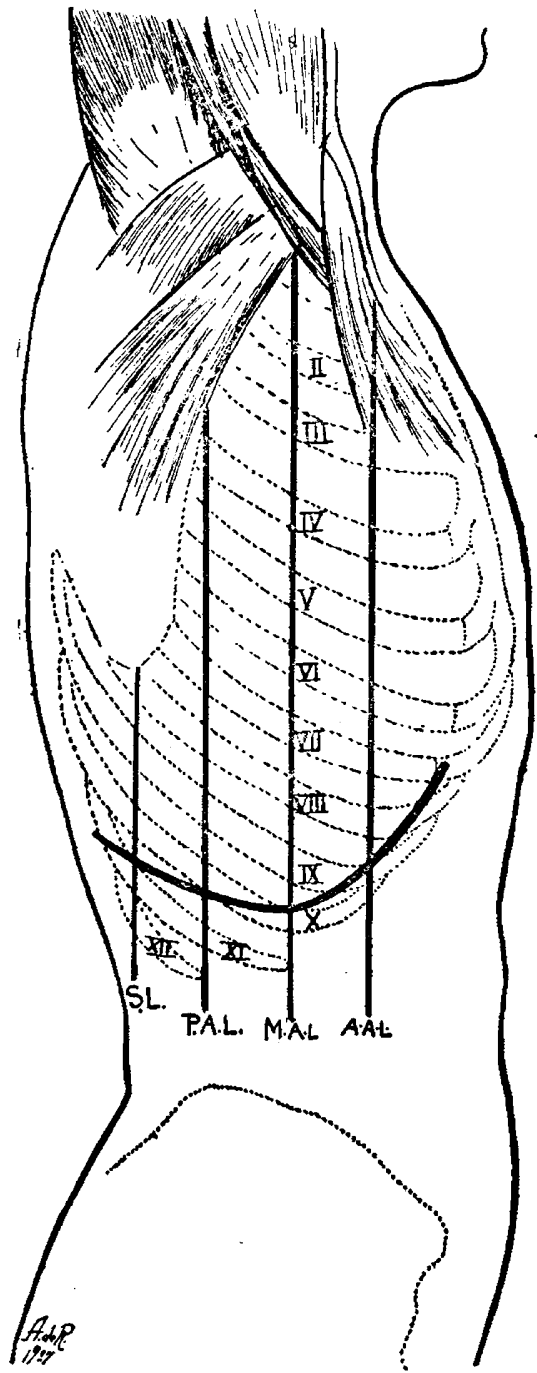

Fig. 2.- Side view of thorax and abdomen to show relation of pleural reflection to ribs and costal cartilages (see description in text). S. L., scapular line. P. A. L., posterior axillary line. M. A. L., midaxillary line. A. A. L., anterior axillary line. The heavy black line represents the level of the reflection of the costal pleura on the diaphragm.

around the appendix. Such cases have been described by Weber, Krohne and Fink. Subphrenic abscess after appendicitis is usually situated in the right side, but 6 left-sided cases have been reported (Schlesinger, Reizenstein, Körte, Darling, and one of my own). Subphrenic abscess seldom occurs as the result of a general suppurative peritonitis. There are only seven such cases on record. It is often impossible to trace any purulent tract from the appendix to the subphrenic region. The position of the appendix bears a direct relation to the formation of the subphrenic abscess. In the majority of cases the appendix was retrocecal (Fig. 3). This is 
the second most frequent normal position of the appendix, according to Morks.

A persistence of the embryonal position of the appendix, due to non-rotation of the cecum, also favors the formation of a subphrenic abscess. The appendix is thus brought in close contact with the right lobe of the liver (Fig. 4). The proximity of the subphrenic spaces to the pleural cavities explains the frequency of pleural effusion as a complication. The pleuritic fluid is usually seropurulent, and may be encapsulated or not. Pericardial involvement is rare, although several cases have been reported in which death followed rupture of the abscess into the pericardium. Munro has called attention to the fact that subphrenic abscess may be the result of a liver abscess after appendicitis. Rupture of the subphrenic abscess into the. lung has been reported in 19 cases. In the majority of cases subphrenic abscesses, after appendicitis, contain only pus. They thus differ from those following perforation of a gastric ulcer, in which the abscess cavity frequently contains both pus and gas.

The anatomic relations of the left subphrenic space are far more complicated than those of the right. The

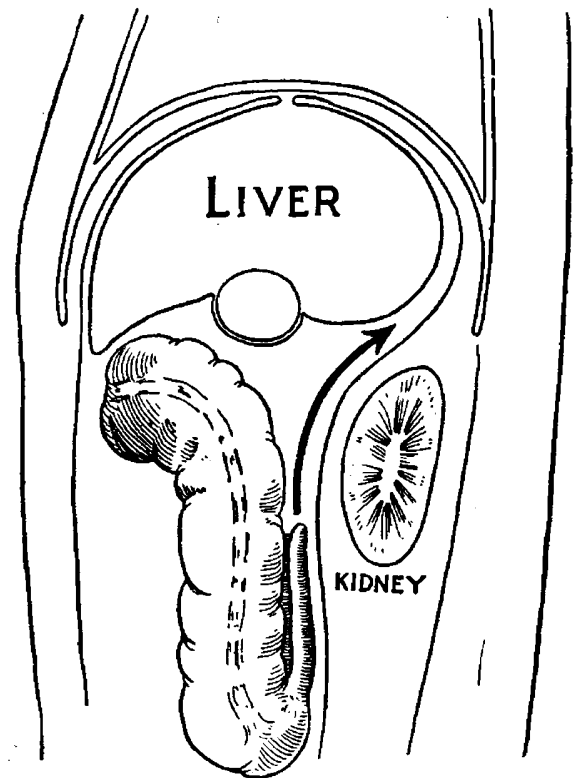

Fig. 3.-Route of infection from a retrocecal appendix to the right subphrenic space (sagittal section).

abscess either lies between the left lobe of the liver, stomach and spleen, or between the stomach and abdominal parietes, or in the lesser omental cavity (Fig. 5).

Before reviewing the symptoms and treatment of this complication of appendicitis, I desire to report the following cases:

CASE 1.-Gangrenous appendicitis, with abscess formation; left-sided subphrenic abscess, diagnosed two weeks after operation; death from septicemia.

F. M., aged 11, was admitted to the Michael Reese Hospital with well-marked symptoms of appendicitis. A well walledoff intraperitoneal abscess eavity was opened, but no effort was made to remove the appendix. Condition quite critical. At the end of the first week the temperature was $106 \mathrm{~F}$., pulse 130 The wound was reopened, but no cause for the septic condition was found. Examination of the thorax on the right side was negative. At the end of twenty-one days an area of dulness was found over the base of the left lower lobe posteriorly. An exploring needle was inserted and fetid pus obtained. The pleural cavity was opened under the impression that the complication was one of abscess of the lung. The left lower lobe was found adherent to the diaphragm. On being separated from the latter a neelle was inserted through the diaphragm and very fetid pus was obtained. An abseess located between the left lobe of the liver and the spleen was drained, by the transpleural route. The septic symptoms improved for a short time, but recurred, and death took place about five weeks after the original operation.

The peculiarity of this case is the left-sided situation of the abscess. The only signs which caused a search for such a complication to be made were the persistence of high fever, rapid pulse and other signs of deep-seated infection, in spite of thorough drainage of the primary appendiceal abscess. The subphrenic infection was not the result of a generalized suppurative peritonitis, since such a condition never existed in this case. The infection probably followed between the adherent coils of intestine along the inner side of the colon to the left subphrenic space (Fig. 1). This case shows the necessity of a thorough physical examination in every case of suspected subphrenic abscess.

CASE 2.-Right-sided subphrenic abscess; acute retrocecal appendicitis; death from subphrenic infection, five days after onset of illness.

H. G., aged 6; seen August 16, 1905 ; history of acute inflammatory affection of right side of abdomen for previous three days. Temperature, $103 \mathrm{~F}$; pulse, 152. Immediate operation. Appendix retrocecal, with tip extending almost to liver. Marked inflammatory changes, and covered with plastic exudate. The appendix was intraperitoneal. There was no perforation, nor evidence of abscess formation. Gauze drainage. Twenty-four hours after operation, pulse rose to 162 , and respirations to 60 .

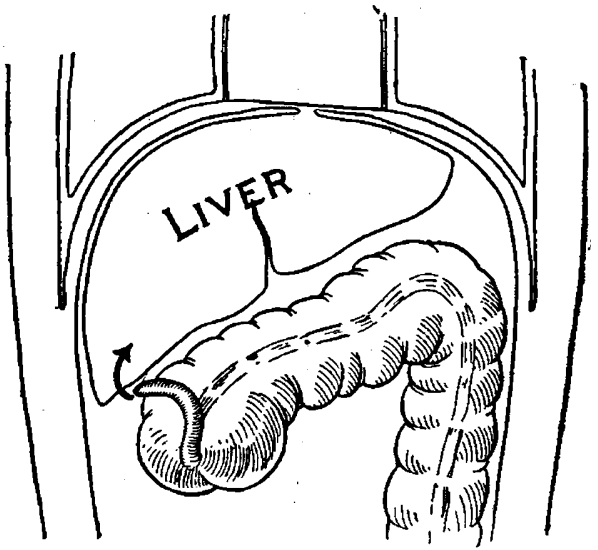
Fig. 4.-Mode of transmission of infection to the right sub-
phrenic space from an appendix which is congenitally displaced phrenle space from an appendix which is congenitally dither in front of or just below the right lobe of the liver.
eithe

Twenty-four hours later, examination of wound negative; no tenderness on palpation over liver, and no area of dulness in lower part of chest. The temperature rose to $104 \mathrm{~F}$., the pulse remaining about 160 . Death occurred sixty hours after operation for removal of appendix, and five days after onset of attack. The autopsy, held three hours after death, showed that there was no free fluid in the peritoneal cavity, and no evidences of any peritonitis. On separating the right lobe of the liver from the abdominal wall a well-encapsulated abscess, containing eight ounces of pus, was found.

The abscess was relatively small and could not be recognized by the ordinary physical signs. The persistence of the fever and rapid pulse should have served as an indication for a systematic exploration of the subphrenic region with the needle had the course been less rapid. The intraperitoneal position of the appendix behind the cecum, with its tip close to the liver, favored the localization of infection in the right subphrenic region. It would be better in such retrocecal appendiceal cases to have the patient placed in the Fowler posi- 
tion after operation, and some method of drainage should be provided for the retrocecal region, making a counter-opening in the loin. There was no abscess formation in the primary appendiceal lesion. The course was a very rapid one.

CASE 3.-Right-sided subphrenic abscess, complicated by encapsulated diaphragmatic empyema. Recovery.

S. F., aged 30, male, was admitted to my service in Cook County Hospital, Sept. 29, 1905, with a diagnosis of empyema. The only history obtainable was that he had ben operated on, one year previously, for appendicitis. A large scar in the right iliac region and the presence of a hernia rendered it possible to infer that an appendiceal abscess had been drained. Two weeks before admission to $\mathrm{my}$ service he was seized with a sharp epigastric pain; temperature on admission, $103 \mathrm{~F}$. There was an area of dulness extending from the eighth rib downward on the right side of the chest in the scapular line, and from the fourth rib in the mammary line. Greatly diminished respiratory and voice sounds were heard over this area.

Exploratory puncture showed thick, fetid pus. The seventh rib was resected in the posterior axillary line; no pus found in the pleural cavity. The right lower lobe was adherent to the diaphragm, and on being separated a considerable quantity of fetid pus escaped. What was supposed to be an encapsulated diaphragmatic empyema was drained, the patient
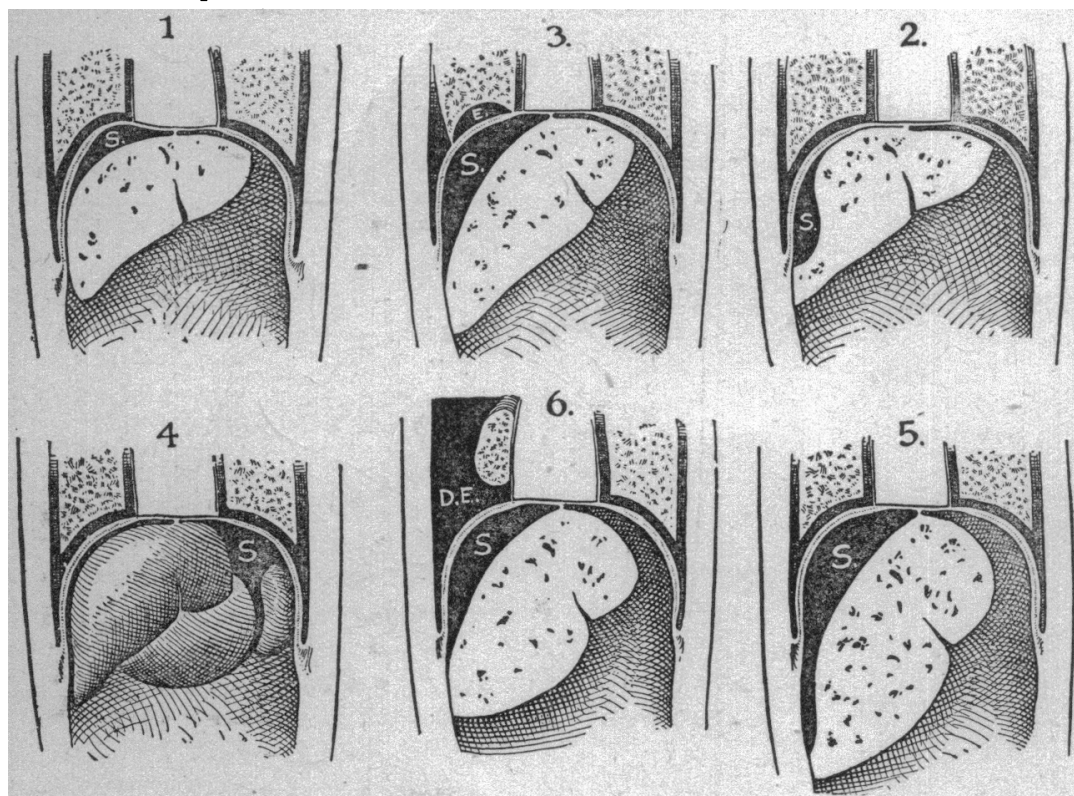

Fig. 5.-Various forms of subphrenic abscesses complicating appendicitis. S., in all of the figures, represents the abscess E., in 3 represents empyema. D. E., in 6, diffuse empyema. 1. Abscess beneath dome of diaphragm. 2. Abscess low down in subphrepic space. 3. Combination of encapsulated diaphragmatic empyema and subphrenic pbrenic spae. 3 . Absom abscess. 4. Left-sided subphrenic abscess. 5. Large abscess occupying entire right sub-
phrenic space. 6. Combination of right subphrenic abscess and non-encapsulated empyema.

showing considerable improvement for a few days, but the septic symptoms recurred, and on October 14, two weeks after admission, the thoracic wound was inspected and a bulging upward of the diaphragm noted. Exploratory puncture showed the presence of a subphrenic abscess, which was drained at its lowest point by resecting the tenth rib in the anterior axillary line. Uneventful recovery.

CASE 4.-Retrocecal appendiceal abscess; subphrenic abscess under right dome of diaphragm; transpleural drainage; postoperative empyema; death from septicemia.

H. A., aged 18; entered my service at the Michael Reese Hospital on Feb. 11, 1906. He had been treated for the previous two weeks for appendicitis. The condition had improved after the first week, but recurrence of fever led his physician to send him to the hospital. On admission, there was rigidity in the right hypochondriac and lumbar regions, and some dulness over the same area. At operation, the ascending colon was found adherent to the abdominal wall along its entire length; a large abscess containing very fetid pus was discov- ered just in front of the right kidney; appendix was retrocecal but intraperitoneal; it was perforated at its middle; after being separated from the posterior aspect of the cecum it was removed. Temperature fell gradually to normal, but rose again five days after opening the appendiceal abscess. The wound was reopened, but no retention found. A counter opening was made in the ileocostal space, without any improvement. Examination of the thorax showed an area of dulness at the convex upper border from the seventh rib downwards, between the anterior axillary and scapular lines.

Exploratory puncture of this area showed the presence of fetid pus. The seventh rib was resected in the posterior axillary line, the needle having been left in place after the exploratory puncture. The needle was found to have entered an abscess containing about six ounces of pus, lying between the upper surface of the liver and the right dome of the diaphragm. It was impossible to suture the diaphragm to the chest wall. The pleural cavity was walled off with gauze, and a drain inserted into the subphrenic abscess across the costophrenic sinus. A very
six days later.

This case illustrates the difficulty of draining those abscesses which are located high up under the dome of

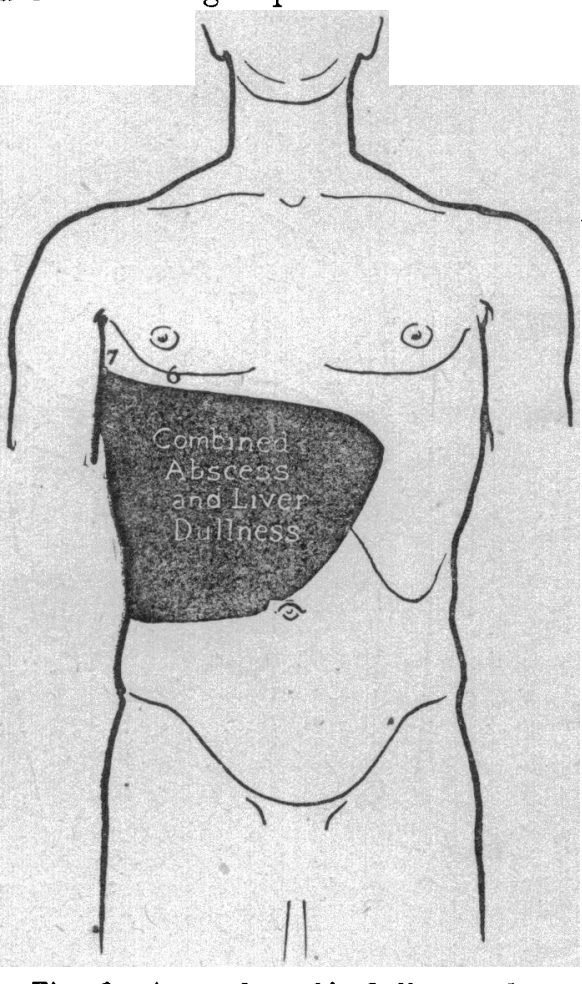

Fig. 6.-Area of combined liver and sub phrenic abscess, dulness in a case of right sided abscess.

the diaphragm. Several of Körte's patients died of a similar postoperative empyema.

CASE 5.-Development of subphrenic abscess during nonoperative treatment of appendicitis. Drainage of abscess while patient extremely septic. Death two weeks after operation.

C. F., aged 38, was seen one month after onset of appendicitis, which had been treated conservatively without operation. On admission the patient was very septic and emaciated with septic nephritis. Rigidity and tenderness over right hypochondriac and epigastric regions. Both right and left lobes of the liver were displaced downward, so that the lower border of the right lobe was below the level of the um. bilicus. There was dulness from the sixth rib downward in the mammary line; from the seventh in the midaxillary line. Some edema over seventh and eighth ribs in the midaxillary line. Absence of respiratory sounds over area of dulness in the axilla. Exploratory puncture through the right seventh interspace in the midaxillary line showed chocolate-colored pus. 
Under local anesthesia, incision was made along the line of the needle left in place. The seventh rib was resected, a distance of about two inches, in the midaxillary line, and immense quantities of pus were evacuated from an ab. scess lying between the posterior surface of the right lobe of the liver, the diaphragm and the upper pole of the kidney. A counter opening was made in the ileocostal space. The initial sepsis continued, and the patient died two weeks after operation.

\section{STMPTOMS.}

The five cases just reported illustrate the varied clinical pictures which a subphrenic abscess produces. There are usually three modes of onset: (a) An acute form, in which the symptoms appear with the attack of appendicitis or a few days afterward. (b) A subacute form, in which signs of infection occur a few weeks after the beginning of the appendiceal attack. (c) A chronic form, in which the symptoms appear months after the appendiceal disease has been recognized or operated on. In Case 3 of my own series the subphrenic symptoms appeared one year after the removal of the appendix.

(a) Acute Form.-This variety may accompany or

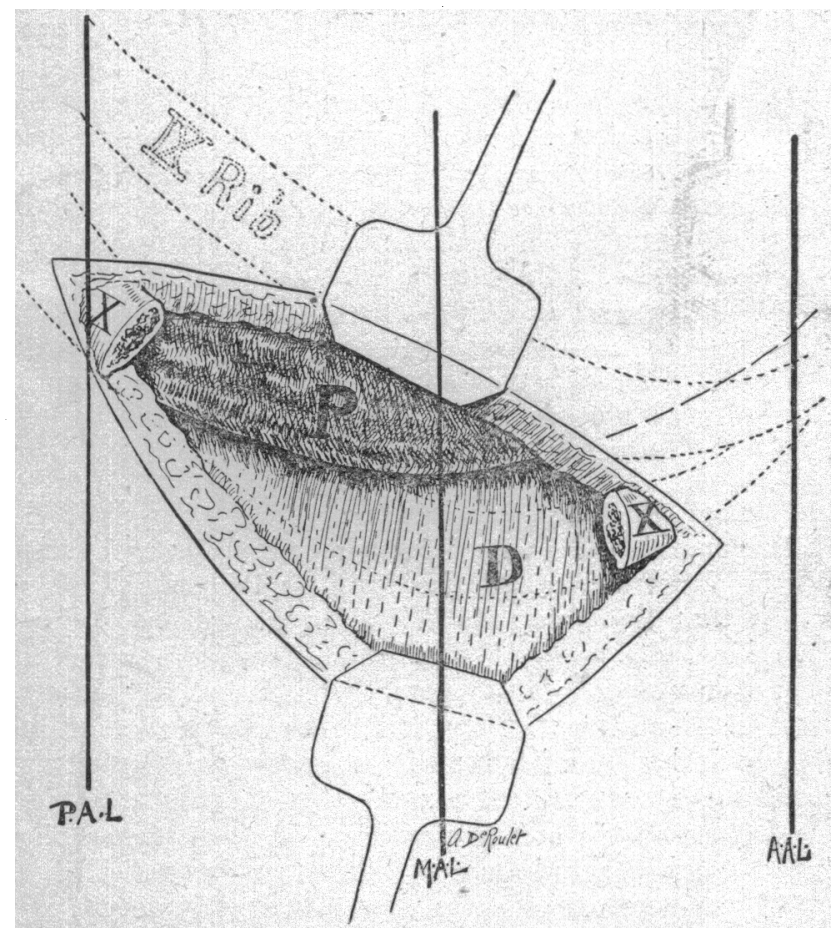

Fig. 7.-Relations of pleural reflection to diaphragm on right side of thorax after resection of tenth rib $x$, between posterior P.. parietal pleura s. L. and anterior axillary line A. A. L. fection on the diaphragm D. M. A. L.. midaxiliary line.

immediately follow an attack of appendicitis which occurs in an appendix arising from a normally placed cecum, or it may be the mode of periappendicular infection, which an abnormally placed appendix takes, as referred to previously. In this latter form the nonrotation of the cecum causes the appendix to lie close to the subphrenic region. Previous articles speak of this mode of onset being accompanied by severe pain in the hypochondrium, by nausea, vomiting and the signs of a severe infection. That pain and the other symptoms just described are not always very marked, is shown in Case 2 of my series.

The persistence of high temperature, with or without accompanying signs of severe septic infection, is the most characteristic symptom of the acute form.
Pain and tenderness in the right hypochondrium may or may not be present:

In some cases physical examination of the chest will be of the greatest aid. If the abscess contains pus only, an area of dulness, with a convex upper border, is found in or near the axillary line (Fig. 6). If the abscess contains gas, there will be an area of tympany above the dulness. It is impossible to differentiate areas of dulness due to pleuritic fluid from those due to a subphrenic abscess, if both are present. If the collection of pus beneath the diaphragm is small, and lies under the dome (Fig. 5), as in Case 3 of my series, or causes but little displacement of the liver, as in Case 2, physical examination is of but little value, and systematic exploratory puncture of the entire hepatic region would be the only means of making a diagnosis. Localized edema and tenderness of the skin of the lower thorax, or close to the costal arch, points to the presence of a subphrenic abscess usually of extraperitoneal location.

In some cases the displacement of the liver downward is very marked. Bulging along the costal margin or in the epigastrium is quite rare. In left-sided abscesses

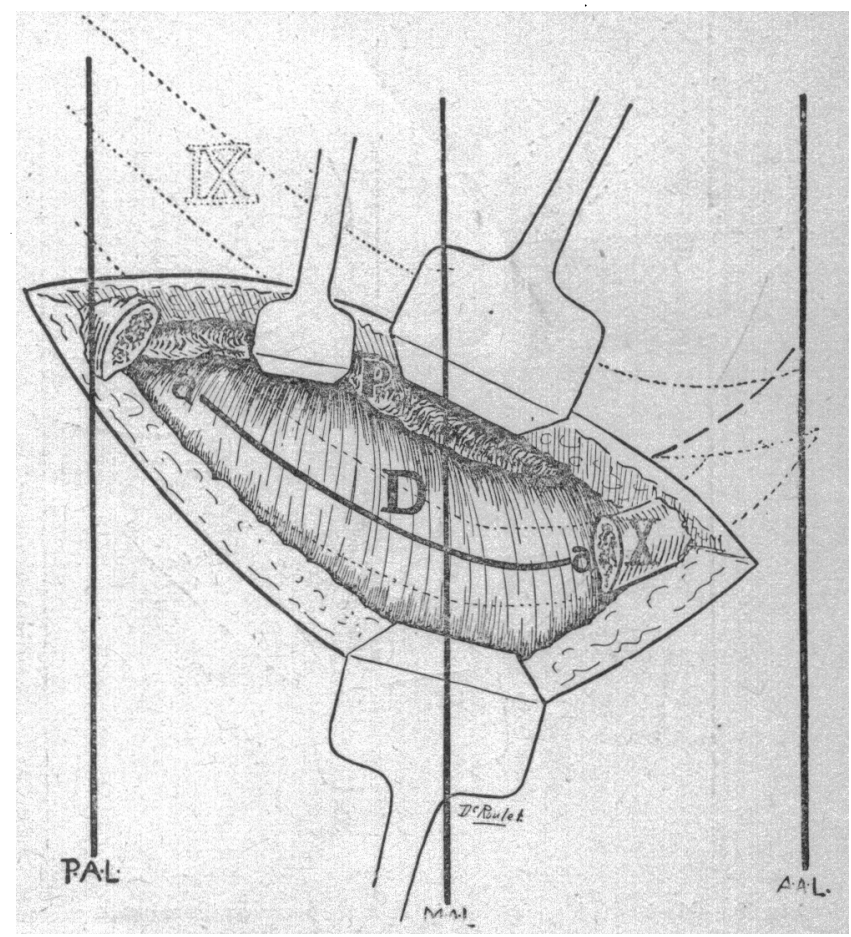

Fig. 8.- Same as Figure 7, but pleural reflection has been retracted upwards and dlaphragm Incised, thus opening the abscess cavity. The line a indicates the line of incision.

there is dulness at the lower portion of the chest, either in front or behind, or on the side. This dulness, if in front, often extends into the epigastrium, or even into the left hypochondriac region.

Exploratory puncture is one of the most valuable means of diagnosis. If the pus is fetid, it is strongly indicative of the presence of a subphrenic abscess. If the needle penetrates quite deeply before pus is obtained, this should also lead to the suspicion of a subphrenic abscess.

If there are marked symptoms of sepsis following an attack of appendicitis, and the exploring needle reveals only a small amount of serous exudate, such a finding should lead one to consider the possible presence of an encapsulated pleuritic exudate over a subphrenic collection of pus. If a non-encapsulated empyema accom- 
panies a subphrenic abscess, it is impossible to differentiate by exploratory puncture unless one first obtains ordinary non-fetid pus, and later, on deeper introduction of the needle, a fetid pus. One can distinguish the pus obtained from an abscess of the liver by the fact that it has no odor and is chocolate-colored.

(b) Subacute Form.-In this form the symptoms appear from eight to twenty-one days after the onset of an attack of appendicitis, or after the removal of the diseased appendix. A week or more after the attack or operation, the temperature rises again, accompanied by gradually increasing symptoms of septic intoxication. The fever is usually of the remittent type, and is accompanied by a corresponding rise in the pulse rate. The patient begins to lose in strength, and becomes greatly emaciated.

The local signs in this form do not differ from those described under the acute mode of onset. In a few cases, this recurrence of fever and of septic symptoms is accompanied by pain over the corresponding side of the chest, cough and dyspnea, but these latter symptoms

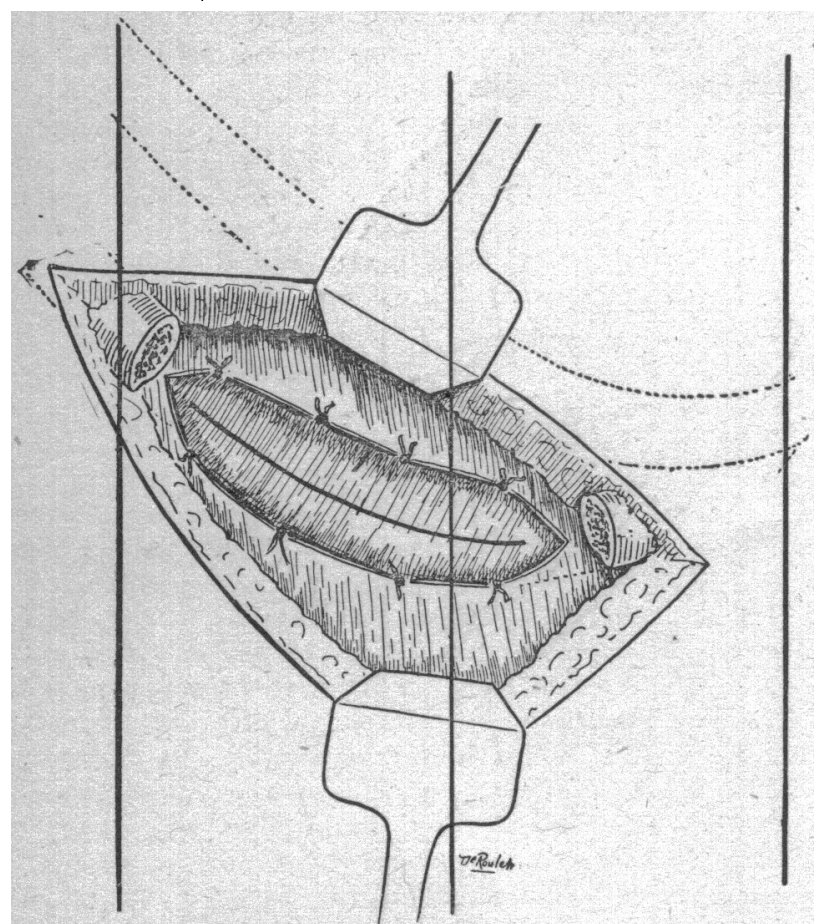

Fig. 9.-Method of operation in low transpleural drainage of subphrenic abscesses. After subperiosteal resection of that portion of the rib which lies over the abscess, the parietal and diaphragmatic layers of the pleura are sutured together.

are not at all constant. In the absence of any retention of pus in or around the seat of operation, this reappearance of fever, ete., should always lead one to search for the physical signs of a subphrenic abscess.

Rupture into the lung has probably taken place if large quantites of pus have been expectorated suddenly in these cases.

(c) Chronic Form.-Case 3 of my own series best illustrates this mode of onset. For weeks to months after an attack of or operation for appendicitis there are indefinite symptoms. In some cases there is an occasional rise of temperature, accompanied by sweats and slight loss of weight and strength. In others, there are no symptoms until the patient is suffering from the symptoms of acute sepsis, due to an obscure deep-seated focus. In these cases the diagnosis can only be made from the local physical signs of dulness, etc., in the lower part of the chest, aided by exploratory puncture, taken in conjunction with the history of an attack of appendicitis, or of an operation for removal of a diseased appendix, or drainage of an appendiceal abscess.

DIAGNOSIs.

If a patient who gives the history of some inflammatory condition in the right lower quadrant of the abdomen, or who has been operated on for appendicitis, has a continued rise of temperature, accompanied by other symptoms of septic intoxication, one should always make a diligent search for a subphrenic abscess. The downward displacement of the liver, the presence of an area of dulness with a convex upper border, continuous with the dulness of the liver (Fig. 6), and the finding of fetid pus on exploratory puncture are very characteristic. If there is localized bulging or edema, the diagnosis is rendered quite easy. The principal condition to be differentiated is empyema. This is, at times, practically impossible. The history of a preceding pneumonia, followed by continued fever, and the local physical signs, is often of great aid in making a diagnosis of empyema. The upper level of the area of dulness is more apt to be horizontal in empyema instead of convex, as in $a$ subphrenic abscess.

In many cases a differentiation is impossible, except from the history and the character of the pus. If the subphrenic abscess contains gas, and the three characteristic zones described under symptoms are present, the diagnosis is not difficult. If an empyema and subphrenic abscess coexist, differentiation is impossible. This is especially true of an encapsulated empyema, as in Case 3. This case also shows that persistence of fever after drainage of an empyema should lead to the suspicion of a subphrenic abscess. The $x$-ray is not sufficiently reliable to be useful as a means of diagnosis.

\section{PROGNOSIS.}

In general, the chances for a spontaneous evacuation of the abscess through the lung or externally are so slight that the prognosis is bad in non-operated cases. Even in those cases of subphrenic abscess in which an operation was done, the mortality has been much greater than it should be. This is due to the fact that the diagnosis has not been made in many cases until the patient is so septic that operation is of little avail.

Elsberg, in his article published in 1901, found that in 73 cases of subphrenic abscess after appendicitis, 11 of 51 patients operated on died, a mortality of 22 per cent. Of 22 patients not operated on, 18 , or 82 per cent., died. The 107 cases which I have been able to collect since the publication of Elsberg's article include - his 73 cases. In these 107 cases (all following appendicitis) the results were as follows:

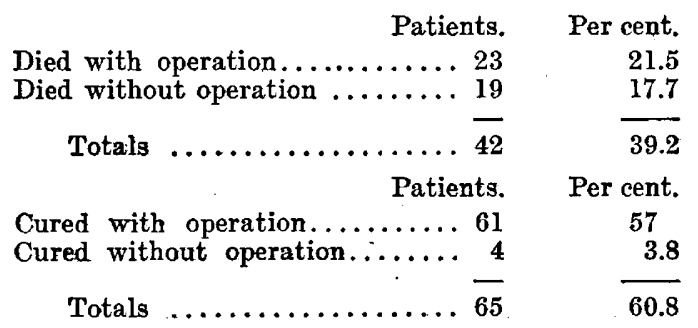

These latter figures show that with the additional 34 cases which I have been able to collect (including my 5) since Elsberg's article, the mortality in 84 cases in which an operation was done was 25 per cent. It is impossible to state what the mortality is in non-operated 
cases, since the majority of these are either not recognized or are not published. 'I'hat it is fully 80 to 85 per cent. must be concluded from the 19 cases which I have been able to collect. When we consider the almost moribund condition in which many of the patients are brought to the surgeon, 75 per cent. of recoveries in 84 operated cases is decidedly encouraging.

If the diagnosis is made at an earlier period of the disease the percentage of recoveries will be far greater than even 75 per cent. Such an early diagnosis can only be made if the physician or surgeon will bear in mind the relative frequency with which subphrenic abscess occurs as a complication of appendicitis.

\section{TREATMENT.}

'There are five methods of draining a subphrenic abscess: (a) By an incision in the epigastrium; (b) by an incision along the costal arch; (c) by an incision in the lumbar region; (d) by the transpleural route; (e) by pushing the pleural reflection upward and opening the abscess cavity through an incision in the diapliragm without opening the pleural cavity. The first three methods are indicated when there is bulging in the epigastrium along the costal arch or in the lumbar region. A simple incision will suffice to evacuate the abscess. If, however, suppuration continues and septic symptoms reappear, it indicates either an accompanying empyema or insufficient drainage, or, finally, a persistence, as Bevan has shown, of the original focus. Such conditions require more extensive operations.

The ideal method of opening a subphrenic abscess, no matter what its origin may be, is by one of the last two methods, viz., resection of ribs over the area of suppuration with or without opening of the pleural cavity.

Before taking up the relative merits and indications of the transpleural and transthoracic but extrapleural routes, I desire to review briefly the anatomy of the pleura in so far as it is of interest in connection with subphrenic abscess. I have found by the dissection of a large number of formalinized cadavers that the majority of text-books on anatomy give the line of reflection of the diaphragmatic pleura at a higher level than is actually the case. Gray gives the lower limits of the pleura, i. e., the line of pleural reflection, as follows: On both sides the line of reflection meets the anterior axillary line at the junction of the eighth rib with its costal cartilage. It continues to descend until it reaches its lowest point at the tenth rib in the midaxillary line.

Cunningham was the first anatomist to call attention to the fact that the line of pleural reflection is lower than was formerly supposed. His observations were confirmed independently by Elsberg and by me. The diaphragmatic pleural reflection as now generally accepted is as follows:

In the mammary line, at the junction of the cartilaginous and bony portions of the eighth rib. Then passing downward (Fig. 2), it follows the lower border of the ninth rib from the anterior to the midaxillary lines, crossing the lower border of the tenth rib at the posterior axillary line, then passing slightly upward again to reach the side of the vertebral column at the level of the twelfth dorsal spine.

One can readily see the value of this knowledge when we recall that the attachment of the diaphragm to the chest wall is lower than the line of the pleural reflection. Elsberg, in his article on "Subphrenic Abscess After Appendicitis," published in 1901, described a method of operating which avoids opening the pleural cavity. He employed this technic in two cases. About two inches of the ninth and tenth ribs are resected in the usual manner, somewhere between the scapula and anterior axillary lines. The pleural reflection is seen in the wound and is pushed upward. An exploring needle is then inserted through the diaphragm and an incision made alongside of the needle, and drainage tubes inserted into the abscess.

In my own work, both on the human subject and on the cadaver, I found that the pleural reflection was so low that one could only resect the tenth rib between the anterior and posterior axillary lines without opening the costophrenic sinus of the pleural cavity. 'The diaphragm lies in the wound and can be incised readily after the pleural reflection has been pushed upward (Fig. 7). If the ninth rib is resected, the costophrenic sinus will certainly be opened unless the resection of the rib is carried out strictly between the anterior and posterior axillary lines and great care is taken in pushing back the periosteum of the ninth rib. This will be understood by referring to Figures 2, 7, 8 and 9 .

If, as in one of my cases, the abscess cavity is situated high up under the diaphragm (Fig. 5), it can only be reached by the transpleural route.

The method described by Elsberg, which I have modified, is the ideal one. It avoids a possible pneumothorax and empyema. In two of Körte's cases and one case of my own the cause of death was postoperative empyema. In many cases it is impossible to open the abscesses by any other route than the transpleural.

In some cases the costophrenic sinus will be found entirely obliterated by adhesions between the costal and diaphragmatic pleure. The pleura can then be incised without opening the pleural cavity proper.

If it is necessary to open the pleural cavity several methods can be employed. If possible, the diaphragmatic pleura should be sutured to the costal pleura. If there is much bulging of the diaphragm it is best to aspirate some of the pus lying beneath it before suturing. If it is impossible to bring the diaphragm to the chest wall the general pleural cavity can either be walled off with gauze or a larger portion of the ninth and tenth ribs can be resected. The former (tamponnade) is very unsatisfactory on account of the danger of leakage. The second method, i. e., more extensive resection, has been recommended warmly by McDill, ${ }^{8}$ who employed it in 100 cases of amebic liver abscess drained by the transpleural route. It had been previously employed by Körte, Lejars and others. After a four-inch partial resection of the ninth and tenth ribs in the midaxillary line an assistant presses the thoracic wall inward against the diaphragm, to which it is sutured, while an incision is made through the diaphragm into the subphrenic abscess.

8. The Jourxal A. M. A., Aug. 10, 1907.

Gelatin Treatment of Pulsating Exophthalmos from Aneurism.-Santos Fernandez reported in the Revista de Med. y Cir., July, 1907, a case of pulsating exophthalmos from traumatic aneurism in which the patient was clinically cured by injections of 100 c.c. of a 1 or 2 per cent. solution of gelatin in a 2 per cent. solution of sodium chlorid. Fifteen injections were made in all between December and August, 1906, and the result was gradual subsidence of the exophthalmos and other symptoms of the aneurism. The case is illustrated, before, during and after treatment. He knows of no other case on record in which an aneurism of the ophthalmic artery was cured under gelatin treatment. 